\title{
Tunable electronic properties of two-dimensional nitrides for light harvesting heterostructures
}

Maria Stella Prete, Adriano Mosca Conte, Paola Gori, Friedhelm Bechstedt, and Olivia Pulci

Citation: Appl. Phys. Lett. 110, 012103 (2017); doi: 10.1063/1.4973753

View online: http://dx.doi.org/10.1063/1.4973753

View Table of Contents: http://aip.scitation.org/toc/apl/110/1

Published by the American Institute of Physics

\section{Articles you may be interested in}

Impact of N-plasma and Ga-irradiation on MoS2 layer in molecular beam epitaxy

Appl. Phys. Lett. 110, 012101012101 (2017); 10.1063/1.4973371

Sub $250 \mathrm{~nm}$ deep-UV AIGaN/AIN distributed Bragg reflectors

Appl. Phys. Lett. 110, 011105011105 (2017); 10.1063/1.4973581

Fermi-level pinning of bilayer graphene with defects under an external electric field

Appl. Phys. Lett. 110, 011601011601 (2017); 10.1063/1.4973426

Millimeter-scale layered MoSe2 grown on sapphire and evidence for negative magnetoresistance

Appl. Phys. Lett. 110, 011909011909 (2017); 10.1063/1.4973519

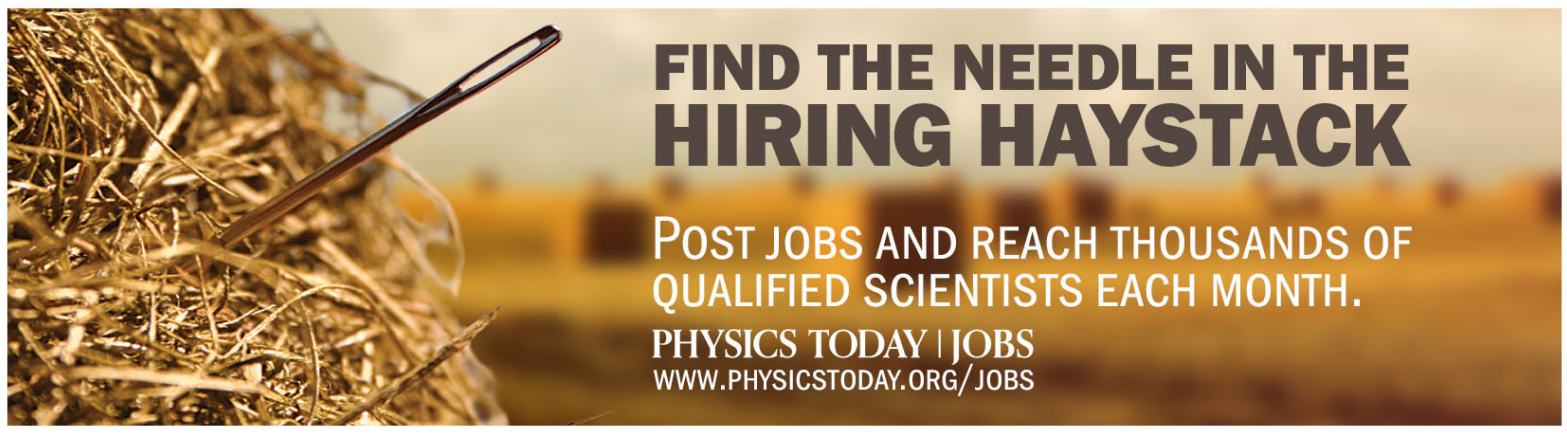




\title{
Tunable electronic properties of two-dimensional nitrides for light harvesting heterostructures
}

\author{
Maria Stella Prete, ${ }^{1}$ Adriano Mosca Conte,${ }^{2}$ Paola Gori, ${ }^{3}$ Friedhelm Bechstedt, ${ }^{4}$ \\ and Olivia Pulci ${ }^{5}$ \\ ${ }^{1}$ Dipartimento di Fisica, Università di Roma 'Tor Vergata', Rome, Italy \\ ${ }^{2}$ Consiglio Nazionale delle Ricerche, CNR-ISMN, and MIFP, Rome, Italy \\ ${ }^{3}$ Engineering Department, Università Roma Tre, Rome, Italy \\ ${ }^{4}$ IFTO, Friedrich-Schiller-Universität and ETSF, Max-Wien-Platz 1, 07743 Jena, Germany \\ ${ }^{5}$ Dipartimento di Fisica, and INFN, Università di Roma 'Tor Vergata', Rome, Italy
}

(Received 2 November 2016; accepted 26 December 2016; published online 5 January 2017)

\begin{abstract}
We study the electronic properties of two-dimensional (2D) group-III nitrides BN, AlN, GaN, InN, and TIN by first-principles approaches. With increasing group-III atomic number, a decrease of the electronic gap from $6.7 \mathrm{eV}$ to $0 \mathrm{eV}$ takes place. $2 \mathrm{D} \mathrm{GaN}$ and $2 \mathrm{D} \mathrm{InN}$ in honeycomb geometry present a direct gap at $\Gamma$, while the honeycomb structures of $\mathrm{BN}$ and AIN tend to be indirect semiconductors with the valence band maximum at $\mathrm{K}$. Alloying of the nitrides allows tuning the gap with cation composition. Interestingly, $\operatorname{In}_{x} \mathrm{Ga}_{1-x} \mathrm{~N}$ and $\operatorname{In}_{x} \mathrm{Tl}_{1-x} \mathrm{~N}$ alloys enable, with varying $x$, to construct type I or type II heterostructures. We demonstrate that it is possible to tailor the electronic and optical response from UV to IR. We suggest that 2D InGaN and InTIN heterostructures may efficiently harvest light and serve as building blocks for a future generation of III-V solar cells. Finally, 2D InTIN with a low In content is eligible as the emitter and detector for $\mathrm{THz}$ applications. Published by AIP Publishing. [http://dx.doi.org/10.1063/1.4973753]
\end{abstract}

The physics of novel two-dimensional (2D) materials, composed of one or several atomic layers, becomes increasingly more interesting because of their extraordinary properties. This has been demonstrated since the discovery of graphene. The fascinating properties and potential applications of $2 \mathrm{D}$ systems have accelerated the search for novel layered materials, such as silicene, ${ }^{1,2}$ germanene, ${ }^{3,4} \mathrm{MoS}_{2},{ }^{5}$ and $\mathrm{BN}^{, 6-8}$ with interesting optical properties. ${ }^{9,10}$ Lowdimensionality effects, as the basis of nano-optics and nanoelectronics, are well recognized to make group-III nitrides interesting for highly efficient light emitting diodes and other optoelectronic devices. 3D nitrides crystallize in wurtzite and zincblende structures. Therefore, preparation of flat honeycomb III-N sheets with graphene-like geometry for $\mathrm{Al}$, $\mathrm{Ga}$, In, Tl-N compounds might be challenging, because presumably no simple route to mechanical or chemical exfoliation can be conceived. The possible existence of stable IIInitrides in 2D single layers is therefore under discussion.

Several examples of successful experimental realization of 2D III-nitrides have been reported in the literature. A monolayer of hexagonal $\mathrm{BN}$ has been deposited on a $\mathrm{Ni}(111)$ surface. $^{6}$ An electronic gap of about $7 \mathrm{eV}$ has been estimated by combining direct photoemission spectroscopy and theoretical calculations. Recently, it has been shown that it is possible to grow nanosheets of hexagonal $\mathrm{AlN}$ on $\operatorname{Ag}(111)$ surfaces. ${ }^{11}$ Ultrathin GaN layers have been grown on InGaN substrates. ${ }^{12}$ Very recently, 2D GaN has been obtained via graphene encapsulation. ${ }^{13}$ From the theoretical side, the stability and the electronic properties of 2D BN, AlN, GaN, and InN have been investigated. ${ }^{14-16}$ The dynamical stability of the corresponding honeycomb crystals with a planar geometry, similar to the one of graphene, has been demonstrated. ${ }^{14}$ However, a better treatment of many-body quasiparticle (QP) effects, similar to what has been done for 2D
$\mathrm{BN},{ }^{14,17-20}$ is required in electronic structure calculations. In this work, we systematically investigate the QP electronic properties of III-N sheet materials, including TIN, in honeycomb graphene-like geometry, in the framework of the Density Functional Theory (DFT) ${ }^{21}$ and Green's function Many Body Perturbation Theory ( $G W$ approximation). ${ }^{22}$ The stability of the flat honeycomb graphene-like structures is predicted. The topological properties of TIN with a vanishing gap are investigated.

For exploring the degree of tunability of their properties, we also study $\operatorname{In}_{x} \mathrm{Ga}_{1-x} \mathrm{~N}$ and $\mathrm{In}_{x} \mathrm{Tl}_{1-x} \mathrm{~N}$ alloys and heterostructures. We find not only tunable gaps with composition but also tunable band discontinuities of nitride heterostructures. Depending on the In or $\mathrm{Ga}$ (or $\mathrm{Tl}$ ) percentage, type I or type II heterostructures may occur. Hence, 2D nitrides represent promising building blocks for nano-optical devices and for efficient solar cells.

We perform DFT calculations using the Quantum Espresso package. ${ }^{23}$ The exchange-correlation potential is approximated within the local-density approximation (LDA). ${ }^{24}$ We use norm-conserving pseudopotentials. The Ga $3 d$, In $4 d$, and $\mathrm{Tl} 5 d$ electrons are treated as valence electrons. This approximation guarantees to find correct structural properties. ${ }^{25}$ In the self-consistent field and total energy calculations a set of $(12 \times 12 \times 1) \vec{k}$ points is used for the Brillouin Zone (BZ) integration. The kinetic energy cut-off of the plane-wave basis set is taken as $100 \mathrm{Ry}$. To prevent interactions between adjacent supercells $21 \AA \AA$ vacuum spacing is kept. To correct the energy bands obtained within DFT, $G_{0} W_{0}$ QP calculations are carried out. $^{22,26}$ Results of $G_{0} W_{0}$ corrections are obtained using $(102 \times 102 \times 1) \vec{k}$-point meshes in the BZ for the exchange part of the self-energy. For the correlation part and for the screened Coulomb interaction $W$, we apply $(51 \times 51 \times 1) \vec{k}$ points and 300 bands. Spurious interactions between adjacent 
TABLE I. Increase $\Delta E_{f o r m}$ of the formation energy per III-N pair, from 3D wurtzite to $2 \mathrm{D}$ honeycomb structures. For BN, $\Delta E_{f o r m}$ refers to layered hexagonal $\mathrm{BN}$. In the second column, we list the lattice constants calculated for the honeycomb geometries. The experimental value for AlN is given in parenthesis. We report the theoretical direct and indirect QP gaps $E_{g}$, and isotropic effective masses $m_{e}^{*}\left(m_{h}^{*}\right)$ of electrons (holes) from Kohn-Sham bands around the $\Gamma$ point. In the last two columns, the electron affinity and the ionization energy are presented. For TIN, SOI corrections are included.

\begin{tabular}{|c|c|c|c|c|c|c|c|}
\hline & $\Delta E_{\text {form }}(\mathrm{eV} /$ pair $)$ & $a(\AA)$ & $E_{g}(\mathrm{eV})$ & $m_{e}^{*}$ (a.u.) & $m_{h}^{*}$ (a.u.) & $\mathrm{A}(\mathrm{eV})$ & $\mathrm{I}(\mathrm{eV})$ \\
\hline $\mathrm{BN}$ & 0.05 & 2.48 & 7.2(KK)/6.7(КГ) & 0.96 & 0.58 & 0.0 & 7.2 \\
\hline AlN & 1.23 & 3.03 (3.13 (Ref. 11)) & $6.5(Г Г) / 5.8(\mathrm{KГ})$ & 0.57 & 1.60 & 1.3 & 7.1 \\
\hline $\mathrm{GaN}$ & 0.98 & 3.15 & 4.1(ГГ)/4.1(КГ) & 0.29 & 0.86 & 2.1 & 6.2 \\
\hline $\mathrm{InN}$ & 1.12 & 3.52 & $1.7(Г Г) / 2.1(\mathrm{~K} Г)$ & 0.10 & 0.43 & 3.7 & 5.4 \\
\hline TIN & 0.86 & 3.67 & 0 & 0.11 & 0.90 & 5.1 & 5.1 \\
\hline
\end{tabular}

supercells have been treated by cutting ${ }^{27}$ the Coulomb potential along $z$ ( $z$ being normal to the sheet material). Since the gap of 2D TIN almost vanishes, but the same happens for QP corrections at the Fermi level, we represent the QP electronic structure of TIN (including spin-orbit interaction (SOI)) by the Kohn-Sham eigenvalues.

For all the 2D nitrides studied, we find that a flat unbuckled honeycomb structure is the most stable geometry, giving rise to local minima on the total energy surface. Their character as metastable phases is indicated in Table I by the positive differences $\Delta E_{\text {form }}$ between their formation energies and those of the bulk wurtzite geometries. The resulting $2 \mathrm{D}$ lattice constants are listed in Table I. They show a clear chemical trend with increasing atomic number of the cation in agreement with other computations (without TIN). ${ }^{14}$ For AlN, the theoretical value tends to underestimate slightly the measured value. ${ }^{11}$ In all cases, the bond lengths in the $s p^{2}$ bonded systems are considerably smaller than in the corresponding $s p^{3}$-bonded bulk nitride crystals.

The electronic band structures are displayed in Figs. 1 and 2. Although the geometry resembles the one of graphene, the electronic properties show striking differences. Because of the different electronegativities between nitrogen and groupIII atoms, a charge transfer occurs from the cation to the anion. It is accompanied by a gap opening, hence no Dirac cone exists. The calculated band gaps are given in Table I, together with the high-symmetry points where the maximum (minimum) of the valence (conduction) band occurs.

With increasing atomic number of the cation, the gap decreases from $6.7(\mathrm{BN})$ to $1.7 \mathrm{eV}(\mathrm{InN})$. Interestingly, at the $G W$ level, the minimum gap of $\mathrm{GaN}$ and $\mathrm{InN}$ turns out to be direct although in $2 \mathrm{D}$ GaN the $\Gamma \Gamma$ and $\mathrm{K} \Gamma$ gaps are practically degenerate. The opposite behavior, an indirect gap, appears for BN and AIN. Spin-orbit splittings of the $p$-derived levels are small in these systems. Even in the case of $\mathrm{InN}$, the splitting is only of the order of $20 \mathrm{meV}^{28}$

As displayed in Fig. 2, a special case is TIN. Without spin-orbit interaction, it turns out to be a zero-gap system. The inclusion of spin-orbit effects produces a gap of $13 \mathrm{meV}$ at $\Gamma$ but the system becomes semimetallic because the uppermost valence band close to $\Gamma$ along the direction $\Gamma \rightarrow \mathrm{K}$, due to wavevector-induced Dresselhaus splitting, is higher than the energy of the lowest conduction band at $\Gamma$ (see Fig. 2). Interestingly, it has been shown that the 3D TIN

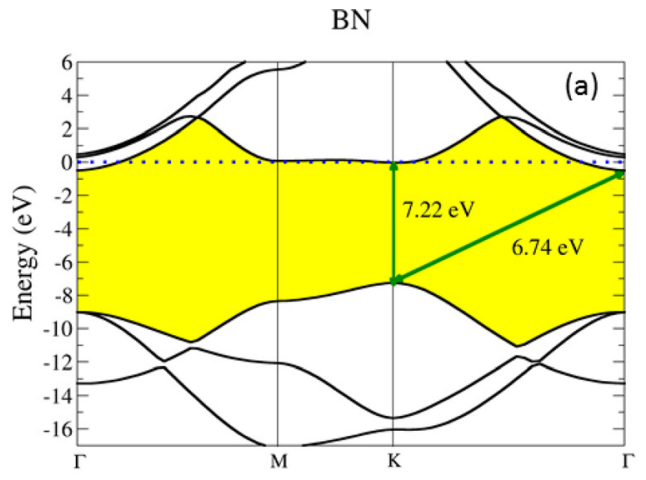

$\mathrm{GaN}$

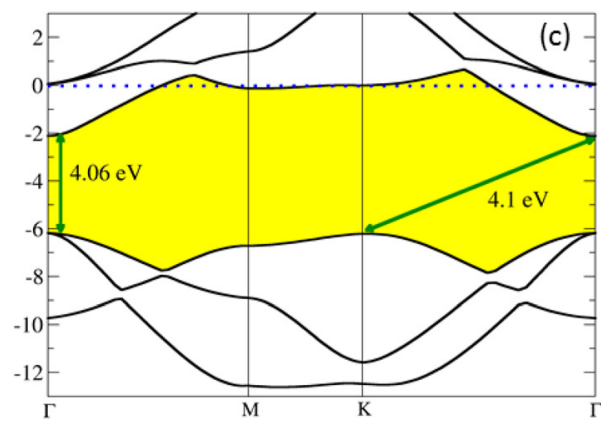

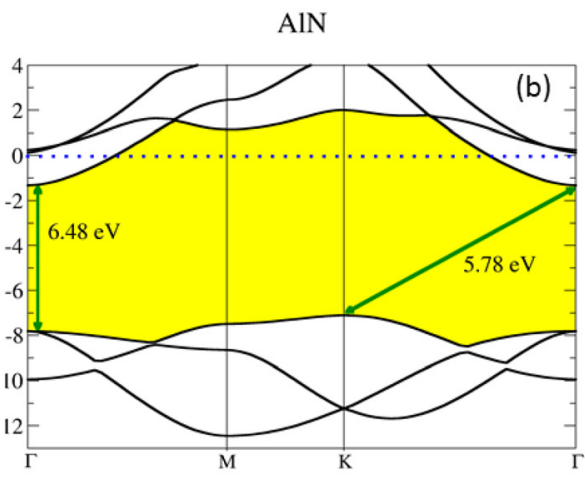

$\mathrm{InN}$

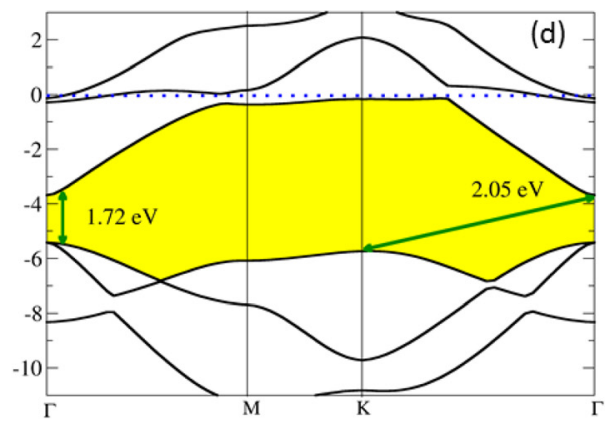

FIG. 1. Electronic band structure of $\mathrm{BN}$ (a), AlN (b), GaN (c), and InN (d) 2D sheets obtained within the GW approximation. The yellow regions characterize the gaps. The vacuum level, identified with the energy zero, is shown as a blue dotted horizontal line. Green arrows indicate direct and indirect gaps. 


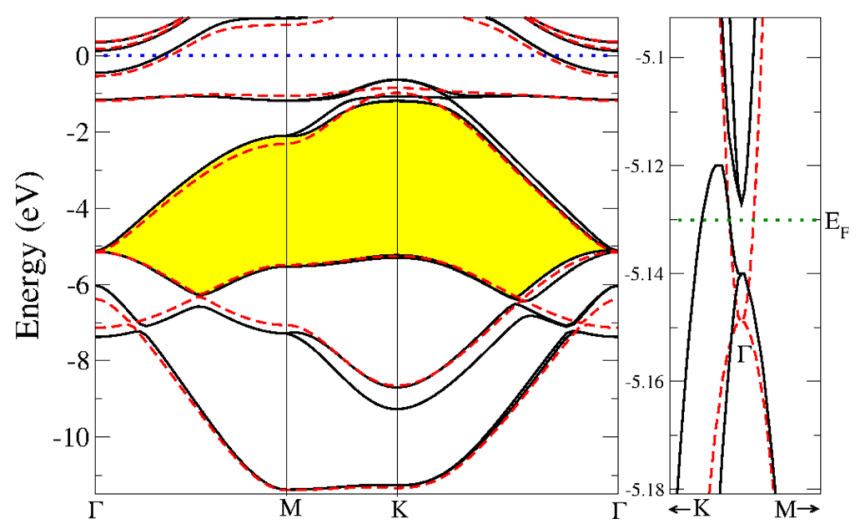

FIG. 2. Left: band structure of TIN with (solid lines) and without (dashed lines) SOI. The blue dotted line indicates the vacuum level. Right: zoom of the bands near the $\Gamma$ point. The high symmetry points are $\mathrm{K}=2 \pi / a(2 / 3,0,0)$ and $\mathrm{M}=2 \pi / a(1 / 2, \sqrt{3} / 6,0)$.

bulk, which crystallizes in the wurtzite structure, ${ }^{29}$ has a zero gap at $\Gamma$, which opens by about $25 \mathrm{meV}$ when SOI is taken into account. ${ }^{30}$ The $3 \mathrm{D}$ system is a topological insulator, while 2D TIN is a Dresselhaus system with spin split parabolas of the valence band along the $\Gamma \mathrm{K}$ direction, but degenerate parabolas along $\Gamma M$ (see Fig. 2).

The effective masses at the high-symmetry $\Gamma$ point are determined by averaging the $\vec{k}$-direction-dependent masses over the two directions $\Gamma \rightarrow \mathrm{M}$ and $\Gamma \rightarrow \mathrm{K}$. These masses are obtained by fitting the energy dispersion of the lowest conduction band (for electron) and of the highest valence band (for hole) to a parabolic function in the vicinity of the $\Gamma$ point. For TIN, we just use the parabola in the $\Gamma \mathrm{M}$ direction. The calculated electron and hole effective masses are also listed in Table I. They show a clear chemical trend, similarly to what happens for the fundamental gap, with the exception of TIN because of the SOI-modified bands. Their trends follow the trend of the fundamental gap. We note that InN has the lightest effective electron mass among the studied 2D III-N systems, as for its 3D counterpart. ${ }^{28}$ The vacuum level is chosen as energy zero in Figs. 1 and 2. For this reason, the conduction band minima $(\mathrm{CBM})$ and valence band maxima (VBM), counted as positive numbers, directly give the electron affinity $\mathrm{A}$ and ionization energy $\mathrm{I}$,

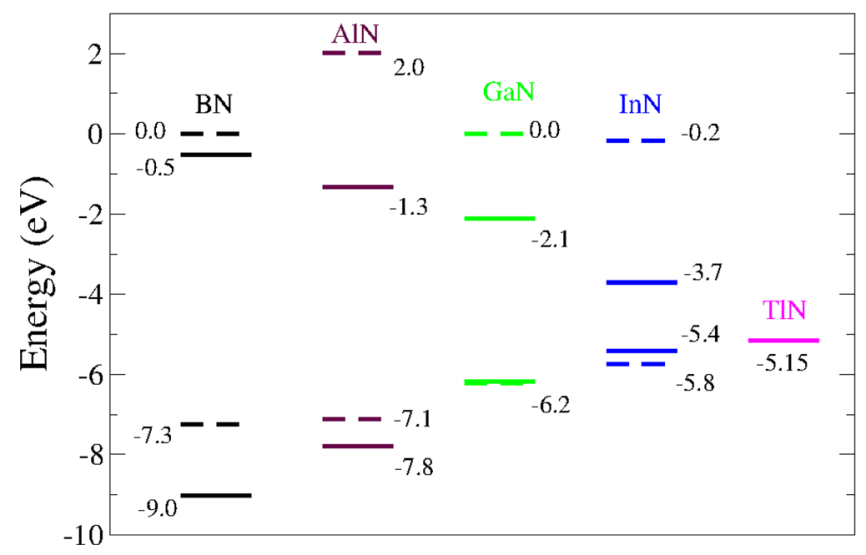

FIG. 3. Energy values of the valence band and conduction band extrema at $\Gamma$ (solid lines) and at $\mathrm{K}$ (dashed lines) of III-N 2D sheets with respect to the vacuum level. respectively. They are also listed in Table I. Along the row $\mathrm{BN}, \mathrm{AlN}, \mathrm{GaN}, \mathrm{InN}$, and TIN, the electron affinity increases from $\mathrm{A}=0 \mathrm{eV}$ to $5.1 \mathrm{eV}$, while the ionization energy I varies between $7.2 \mathrm{eV}$ and $5.1 \mathrm{eV}$.

The 2D III-nitride sheets can be combined in bilayer heterostructures or even vertical superlattices. Then, the selection of the material components appears as an additional tuning parameter, which affects the heterostructure type and the accompanying electron and/or hole confinement. We characterize such heterosystems by the natural band discontinuities, shown in Fig. 3. They have been derived by the vacuum-level alignment. This figure clearly shows the type-I character of the band alignments at the AIN/GaN, GaN/InN, and InN/TIN heterointerfaces. However, also the BN/AIN interface exhibits a type-I heterostructure character if band extrema at different high-symmetry points are considered. Considering the small (with the exception of holes in AlN) effective electron and hole masses listed in Table I, strong confinement effects are expected for TIN (InN, GaN) quantum wells consisting of one atomic layer embedded by $\mathrm{InN}$ ( $\mathrm{GaN}, \mathrm{AlN})$ barrier materials.

In order to add a degree of freedom in the possible tuning of the gaps and of the band discontinuities, we also study alloys of $\mathrm{GaN}$ and $\mathrm{InN}$ as well as $\mathrm{InN}$ and TIN. Thereby, we model the alloys for $x=0.25,0.50$, and 0.75 by $2 \times 2$ cells with 8 atoms, i.e., with one, two, or three cations of one element in the unit cell. The geometries are optimized and used to compute the electronic structures. The $G W$ corrections are obtained according to a linear interpolation between the QP corrections of the end components. We verify that for both alloys the lattice parameter and the band gap vary with the composition, somewhat non linearly. The two systems have a direct gap at $\Gamma$. In particular for $\operatorname{In}_{x} \mathrm{Ga}_{1-x} \mathrm{~N}$, as $x$ increases, the gap ranges from UV to visible. For $\operatorname{In}_{x} \mathrm{Tl}_{1-x} \mathrm{~N}$, we can tune the gap from the visible frequency range for $x=1$ to the $\mathrm{THz}$ regime for $x<0.25$.

The detailed results are illustrated in Fig. 4. The positions of the CBM and VBM indicate the variation of the band edges
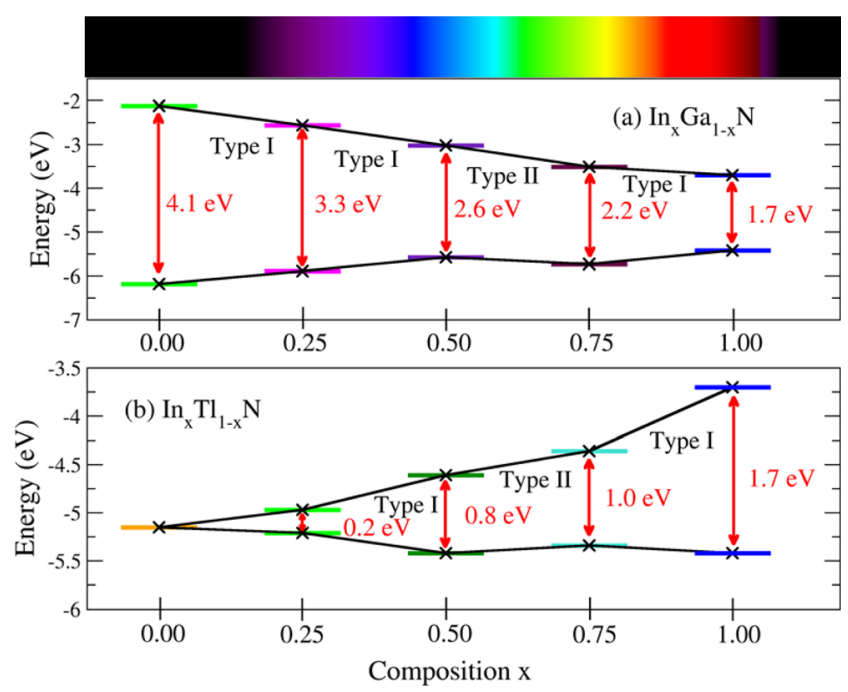

FIG. 4. Direct band gap and band extrema with respect to the vacuum level (used as energy zero) of $\operatorname{In}_{x} \mathrm{Ga}_{1-x} \mathrm{~N}$ (a) and $\mathrm{In}_{x} \mathrm{Tl}_{1-x} \mathrm{~N}$ (b). The spectrum in (a) indicates the variation of the absorption edge in InGaN with composition from the UV to the visible spectral range. 
and the fundamental gap with the composition. The strongest effect comes from the conduction band, since this is related to the cations. Both alloys, $\operatorname{In}_{x} \mathrm{Ga}_{1-x} \mathrm{~N}$ and $\operatorname{In}_{x} \mathrm{Tl}_{1-x} \mathrm{~N}$, exhibit a bowing of the fundamental gaps. The bowing parameters $\mathrm{b}=1.32 \mathrm{eV}$ for $\operatorname{In}_{x} \mathrm{Ga}_{1-x} \mathrm{~N}$ and $\mathrm{b}=0.24 \mathrm{eV}$ for $\mathrm{In}_{x} \mathrm{Tl}_{1-x} \mathrm{~N}$ are of the order of magnitude of 3D III-N nitrides, at least for InGaN. ${ }^{31}$ We also compare the band alignments of these systems to identify the type of heterostructure for an interface between two sheets with different compositions. Interestingly, the heterostructure type changes from type I to type II for intermediate compositions, thus showing 2D III-N nitrides to be reliable material candidates for electron-hole separation in efficient solar cells. The reason is the non-linear variation of the valence-band discontinuities with composition as a consequence of the drastic changes of the electrostatics with the ratio of the two cations in the unit cell. An additional reason for the surprising band alignment of alloys near compositions $x=0.5$ is related to the fact that for this value the strain, in the alloys, is maximum.

To summarize, in this work, using a combination of the $a b$-initio density functional theory for the ground-state properties and $G W$ approximation for the excited state properties, we have calculated the lattice parameters and the band structures of 2D nitrides crystallizing in flat honeycomb geometries. These 2D materials show a wide spread of lattice constants and band gaps. We have shown that, with increasing group-III atomic number, i.e., with increasing covalent radii, the $2 \mathrm{D}$ lattice constants increase up to $50 \%$ while the gaps decrease up to almost $100 \%$. Going from $\mathrm{BN}$ to $\mathrm{InN}$, the band gap ranges from UV to visible. The only exception is that TIN, at the DFT level, is a semimetallic system with extremely small hole (electron) pockets at the $\Gamma \mathrm{K}$ lines $(\Gamma$ point). Important spin-orbit effects are found in $2 \mathrm{D} \mathrm{TlN}$, which produce a few meV gap at $\Gamma$, making it (when some degree of alloying with InN is considered) an appealing material for $\mathrm{THz}$ applications. We also show that $2 \mathrm{D}$ InN has the lightest effective mass among the studied III-N compounds. Therefore, strong confinement effects are predicted for InN embedded by $\mathrm{GaN}$, even using their layered polymorphs. Our study of 2D nitride heterostructures and alloys points out that type I and type II heterostructures are possible to form and that mixing GaN and TIN with InN can tune the gap from UV to IR and even to the $\mathrm{THz}$ regime.

We acknowledge EU support within the HORIZON2020 RISE CoExAN (GA.644076). Computer time was granted by ENEA CRESCO and CINECA.
${ }^{1}$ P. Vogt, P. de Padova, C. Quaresima, J. Avila, E. Frantzeskakis, M. C. Asensio, A. Resta, B. Ealet, and G. Le Lay, Phys. Rev. Lett. 108, 155501 (2012).

${ }^{2}$ Z.-L. Liu, M.-X. Wang, C. Liu, J.-F. Jia, P. Vogt, C. Quaresima, C. Ottaviani, B. Olivieri, P. de Padova, and G. Le Lay, APL Mater. 2, 092513 (2014).

${ }^{3}$ Y. Fukaya, I. Matsuda, B. Feng, I. Mochizuki, T. Hyodo, and S. Shamoto, 2D Mater. 3, 035019 (2016).

${ }^{4}$ L. Zhang, P. Bampoulis, A. von Houselt, and H. J. W. Zandvliet, Appl. Phys. Lett. 107, 111605 (2015).

${ }^{5}$ B. Radisavljevic, A. Radenovic, J. Brivio, V. Giacometti, and A. Kis, Nat. Nanotechnol. 6, 147-150 (2011).

${ }^{6}$ A. Nagashima, N. Tejima, Y. Gamou, T. Kawai, and C. Oshima, Phys. Rev. B 51, 4606 (1995).

${ }^{7}$ K. S. Novoselov, D. Jiang, F. Schedin, T. Boot, V. V. Khotkevich, S. Morozov, and A. K. Geim, Proc. Natl. Acad. Sci. U.S.A. 102, 10451 (2005).

${ }^{8}$ B. J. Feng, J. Zhang, Q. Zhong, W. Li, S. Li, H. Li, P. Cheng, S. Meng, L. Chen, and K. Wu, Nat. Chem. 8, 563-568 (2016).

${ }^{9}$ L. Matthes, O. Pulci, and F. Bechstedt, New J. Phys. 16, 105007 (2014); O. Pulci, P. Gori, M. Marsili, V. Garbuio, R. Del Sole, and F. Bechstedt, Europhys. Lett. 98, 37004 (2012).

${ }^{10}$ M. Palummo, G. Giorgi, L. Chiodo, A. Rubio, and K. Yamashita, J. Phys. Chem. C 116(34), 18495-18503 (2012).

${ }^{11}$ P. Tsipas, S. Kassavetis, D. Tsoutsou, E. Xenogiannopoulou, E. Golias, S. A. Giamini, C. Grazianetti, D. Chiappe, A. Molle, M. Fanciulli, and A. Dimoulas, Appl. Phys. Lett. 103, 251605 (2013).

${ }^{12}$ S. Alamiè, A. Navarro Quezada, D. Skuridina, C. Reich, D. Henning, M. Frentrup, T. Wernicke, I. Koslow, M. Kneissl, N. Esser, and P. Vogt, Mater. Sci. Semicond. Process. 55, 7-11 (2016).

${ }^{13}$ Z. Y. Al Balushi, K. Wang, R. K. Ghosh, R. A. Vilá, S. M. Eichfeld, J. D. Caldwell, X. Qin, Y.-C. Lin, P. A. DeSario, G. Stone et al., Nat. Mater. 15, 1166 (2016).

${ }^{14}$ H. Sahin, S. Cahangirov, M. Topsakal, E. Bekaroglu, E. Akturk, R. T. Senger, and S. Ciraci, Phys. Rev. B 80, 155453 (2009).

${ }^{15}$ A. Onen, D. Kecik, E. Durgun, and S. Ciraci, Phys. Rev. B 93, 085431 (2016).

${ }^{16}$ H. L. Zhuang, A. K. Singh, and R. G. Hennig, Phys. Rev. B 87, 165415 (2013).

${ }^{17}$ N. Berseneva, A. Gulans, A. V. Krasheninnikov, and R. M. Nieminen, Phys. Rev. B 87, 035404 (2013).

${ }^{18}$ F. Huser, T. Olsen, and K. S. Thygesen, Phys. Rev. B 87, 235132 (2013).

${ }^{19}$ L. Wirtz, A. Marini, and A. Rubio, Phys. Rev. Lett. 96, 126104 (2006).

${ }^{20}$ M. Topsakal, E. Akturk, and S. Ciraci, Phys. Rev. B 79, 115442 (2009).

${ }^{21}$ W. Kohn and L. J. Sham, Phys. Rev. A 140, A1113 (1965).

${ }^{22} \mathrm{~F}$. Bechstedt, Many-Body Approach to Electronic Excitations (Springer, Berlin, 2015).

${ }^{23}$ See http://www.quantum-espresso.org/ for DFT details of the numerical approaches and the of the code.

${ }^{24}$ J. P. Perdew and A. Zunger, Phys. Rev. B 23, 5048 (1981).

${ }^{25}$ A. F. Wright and J. S. Nelson, Phys. Rev. B 51, 7866 (1995).

${ }^{26}$ See www.etsf.eu for the GW code CHISIG developed within the ETSF network.

${ }^{27}$ C. A. Rozzi, D. Varsano, A. Marini, E. K. U. Gross, and A. Rubio, Phys. Rev. B 73, 205119 (2006).

${ }^{28}$ L. de Carvalho, A. Schleife, and F. Bechstedt, Phys. Rev. B 84, 195105 (2011).

${ }^{29}$ N. Saidi-Houat, A. Zaoui, and M. Ferhat, J. Phys.: Condens. Matter 19, 106221 (2007).

${ }^{30}$ X.-L. Sheng, Z. Wang, R. Yu, H. Weng, Z. Fang, and X. Dai, Phys. Rev. B 90, 245308 (2014).

${ }^{31}$ L. de Carvalho, A. Schleife, J. Furthmuller, and F. Bechstedt, Phys. Rev. B 85, 115121 (2012). 